\title{
Estimation of the Thermal and Photochemical Stabilities of Pheromones
}

F. N. Tomilin ${ }^{1,2}$, A. S. Fedorov ${ }^{1,2}$, P. V. Artyushenko ${ }^{3}$, S. G. Ovchinnikov ${ }^{1,2}$, T.M. Ovchinnikova ${ }^{4}$, P. E. Tsikalova ${ }^{3}$, and. V G. Soukhovolsky ${ }^{4}$.

${ }^{1}$ Kirensky Institute of Physics, Federal Research Center KSC SB RAS, Krasnoyarsk, 660036 Russia

${ }^{2}$ Siberian Federal University, Krasnoyarsk, 660041 Russia

${ }^{3}$ Federal Research Center KSC SB RAS, International Scientific Center for Extreme Organism

States Research, Krasnoyarsk, 660036 Russia

${ }^{4}$ Sukachev Institute of Forest, Federal Research Center KSC SB RAS, Krasnoyarsk, 660036 Russia

^e-mail:felixnt@gmail.com

\begin{abstract}
The correlation between the kinetic stability of molecules against temperature and variations in their geometric structure under optical excitation has been investigated by the example of different organic pheromone molecules sensitive to temperature or ultraviolet radiation using the density functional theory. The kinetic stability has been determined by the previously developed method based on the calculation of the probability of extension of any structural bond by a value exceeding the limit value $\mathrm{L}_{\text {мax }}$ corresponding to the breaking of the bond under temperature excitation. The kinetic stability calculation only requires the eigenfrequencies and vibrational mode vectors in the molecule ground state to be calculated, without determining the transition states. The weakest bonds in molecules determined by the kinetic stability method have been compared with the bond length variations in molecules in the excited state upon absorption of light by a molecule. Good agreement between the results obtained is demonstrated and the difference between them is discussed. The universality of formulations within both approaches used to estimate the stability of different pheromone molecules containing strained cycles and conjugated, double, and single bonds allows these approaches to be applied for studying other molecules.
\end{abstract}

Key words: kinetic stability, atomic structure, density functional theory, excited states, terpenes. 


\section{Introduction}

The transmission of information by insects is carried out with the help of special odorous substances called pheromones[1]. For most insects pheromones consist of several components, belonging to different classes of substances. For example, moths of the family Geometridae of the insect order Lepidoptera use as pheromone unsaturated straight-chain hydrocarbons obtained 17-21 carbon atoms or their oxygen-containing derivatives (fig. $1 \mathrm{~A}$ ), for xylophagous insects of the order Coleoptera most of the compounds belong to the group of terpenes and terpenoids (fig. 1B).

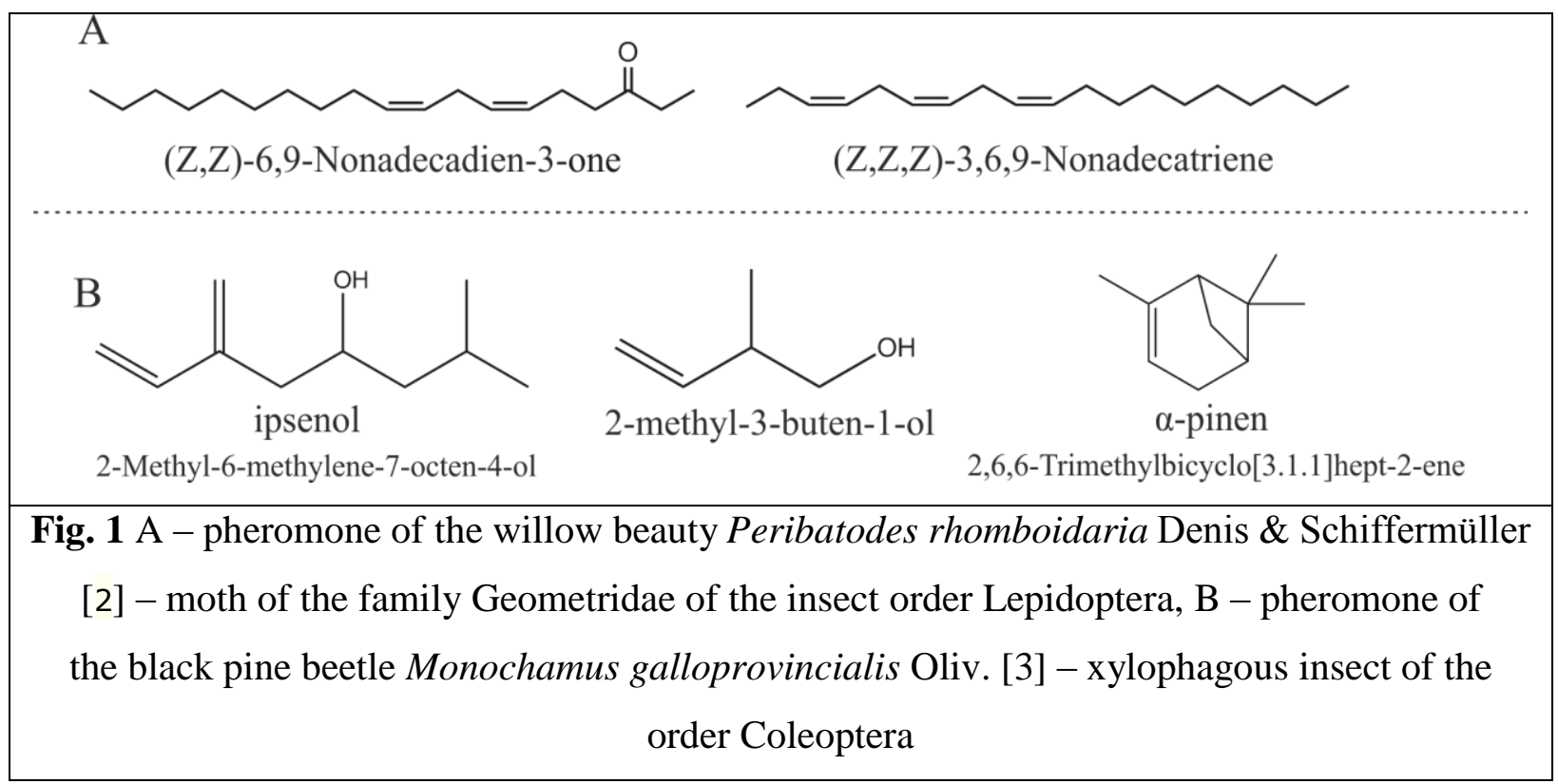

The stability of pheromone molecules, i.e., their lifetime under the action of external factors, including irradiation, heating, and chemical interaction with air components, determines, to a great extent, the efficiency of insect chemical communication. The pest insect population fecundity on the one hand, and the beneficial insect population decline on the other hand, determine the importance of universal theoretical methods for estimating the effect of external factors on insect chemical communication based on recognition of pheromone molecules by insects of the opposite sex before the decay of such molecules under ultraviolet (UV) optical irradiation and heating. Due to the huge diversity of insect species using multicomponent mixtures of organic compounds as pheromones, the lifetime of such molecules should be estimated using theoretical techniques [4-9], since the experimental investigations of the properties of pheromone molecules known to date are nearly impossible.

In [9], we studied the effect of humidity and light on pheromone molecules of xylophage insects using the density functional theory (DFT) calculations based on the B3LYP method. In the present work, we estimate the temperature stability of pheromone molecules using the nanostructure kinetic stability (KS) technique proposed by us in [10-11] and successfully 
applied for estimating the KS and experimental yield of molecules of different fullerenes and carbon nanotubes [10] and simple saturated hydrocarbon molecules [11]. The insect pheromone molecules, which have a limited lifetime and are easily destructed by external factors, can serve as references for testing our KS calculation technique. To perform the complex estimation of the stability of different pheromone classes against temperature and light, we compared the probabilities of breaking of the weakest bonds in molecules and their lengths calculated using the KS technique, as well as the variations in the geometry of molecules under light excitation.

\section{Experimental}

Here, we employ our theoretical kinetic stability (KS) model, which was proposed first and applied to studying some fullerenes and carbon nanotubes in [10]. The model is based on calculating the probability of nanostructure bond breaking via determining the normal vibrational modes and using the central limit theorem. The main features of the initial KS model were described in detail in the Supporting Information, Part S1. The normal vibrational modes were found using the force matrix calculations and the subsequent calculations of all vibrational eigenvalues and eigenvectors. According to the KS model, the probability $\mathrm{P}_{\text {destr }}$ of the molecule weakest bond breaking corresponds to the molecule lifetime: the high probability is indicative of the short lifetime of the molecule and vice versa. Alternatively, the high or low value of the inverse quantity $\mathrm{P}_{\text {destr }}^{1}$ is indicative of the high or low molecule's lifetime, respectively.

Here, we only point out that the KS is based on the assumption that the extension of any chemical bond connecting two atoms is a joint result of the effect of a great number of vibrational modes, with each mode make a minor contribution to the displacement of the atoms connected by the bond. Under these conditions, it is reasonable to use the central limit theorem (CLT) of statistics. Using the CLT, we can calculate the probability of the bond breaking $\mathrm{P}_{\text {destr }}$ when the bond between atoms $n$ and $m$ is broken if the change of bond length $\Delta \vec{R}(n, m, t)>X_{\max }$, where $X_{\max }$ is the maximum bond length. At that the probability of the bond breaking can be written as:

$$
P_{\text {destr }}=P\left(\Delta R(n, m, t) \geq X_{\max }\right)=\frac{2}{\sqrt{\pi} s_{n}(n, m)} \int_{X_{\max }}^{\infty} e^{-\frac{u^{2}}{2 s_{n}^{2}(n, m)}} d u=1 / 2 \operatorname{erfc}\left(\frac{X_{\max }}{\sqrt{2} s_{n}(n, m)}\right),
$$

where $\operatorname{erfc}(\mathrm{x})=1-\operatorname{erf}(\mathrm{x}), \operatorname{erf}(\mathrm{x})$-error function, and the total variance $s_{N}^{2}(n, m)=\sum_{k=1}^{n} \sigma_{k}^{2}(n, m)$ for $(\mathrm{n}, \mathrm{m})$ pair is the sum of variances over all the vibrational modes $k$, which can be easily calculated using the vibrational mode eigenvector $k$ and eigenvalues and temperature $T$. 
The maximum chemical bond extension in all the calculations was taken to be $\mathrm{L}_{\text {мах }}=\mathrm{X}_{\text {мах }}{ }^{-}$ $\mathrm{L}_{0}=0.98 \mathrm{~A}$, where $\mathrm{L}_{0}$ is the equilibrium bond length. This value $\mathrm{L}_{\text {мах }}$ was determined in our work [10] devoted to the KS calculations of some small linear hydrocarbon and benzene molecules at high temperatures. The $\mathrm{L}_{\text {мах }}$ value was determined by fitting of the molecule total energy (U(r)) profile under the bond extension by the quadratic function. The KS values predicted by our model were found to be qualitatively consistent with the relative experimental abundance of some fullerenes and nanotubes of different chiralities. Here, we present the KS model, which was applied to some pheromone molecules.

The pheromone molecule geometry was optimized by the quantum chemical DFT technique using the B3LYP method [12-14] in the cc-pVDZ basis of the GAMESS program [15]; after that, the KS was analyzed via calculating the Hessian for the molecule ground state. To estimate the most probable changes in the geometry of molecules under photoexcitation, we optimized the geometry in the excited state by the time-dependent TD/B3LYP/cc-pVDZ technique [16-17]. The B3LYP functional is one of the most popular; it yields good agreement between the calculated parameters of organic compounds and the experiment [18-21]. The correlation consistent cc-pVDZ basis set allows one to correctly describe bond lengths, valence angles, dipole moment, electron density distribution, and other structural and spectral characteristics of organic molecules [22-23].

The structure of natural molecules can be affected by temperature and solar radiation. The terpene molecules exposed to the UV radiation can pass to the excited state; in this case, the bond lengths and valence angles in molecules change, the bonds can break, and the molecular cycles can open. After that, the modified molecules can further transform to a new substance [24]. The stability of molecules against various external factors can be estimated using the approach based on quantum-mechanical calculations.

Most compounds belong to the terpene and terpenoid groups [25-27] related to the resin odorous substances. The species specificity of terpenes is determined by the structural and optical isomers. The geometrical parameters of molecules (Fig. 2) do not allow atoms to freely exchange by their positions and, consequently to form different conformers. For the investigations, we chose pheromones of three xylophage insect species, which are dangerous for forest, specifically, bark beetles Ips typographus L., black fir tree beetles Monochamus urussovi Fish., and black pine beetles Monochamus galloprovincialis Oliv. belonging to the Coleoptera order. The complex and diverse structure of the Coleoptera pheromones (Fig. 2) allowed us to analyze the applicability of the KS technique for different chemical compounds. 


\section{Ips typographus}

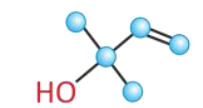

2-methyl-3-buten-2-ol

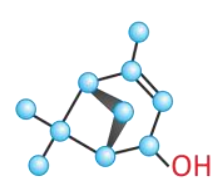

cis-verbenol

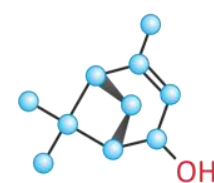

trans-verbenol

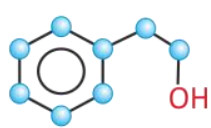

2-phenylethanol

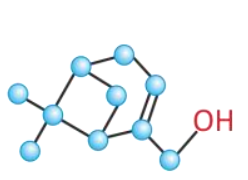

myrtenol

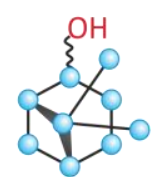

trans-myrtenol

\section{Monochamus urussovi}

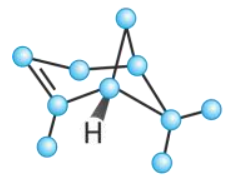

$1 \mathrm{~S}-\alpha$-pinene

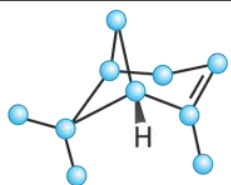

1R- $\alpha$-pinene

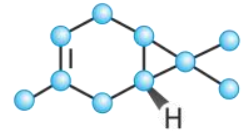

$3 S$-carene

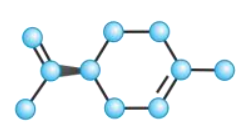

R-limonene

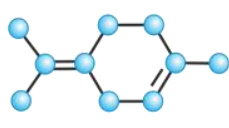

$\alpha$-terpinolene

Monochamus galloprovincialis

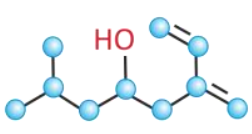

ipsenol

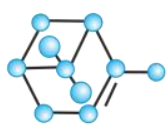

$\alpha$-pinene

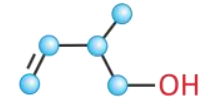

2-methyl-3-buten-1-ol

Fig. 2 I.typographus, M.urussovi, and M.galloprovincialis xylophage insect pheromones.

Carbon atoms are marked in blue

The Ips typographus pheromone contains 2-methyl-3-buten-2-ol, cis-verbenol, transverbenol, 2-phenyl-ethanol, myrtenol, and trans-myrtenol. The Monochamus urussovi pheromone contains five substances without oxygen-bearing ones: 1S-alpha-pinene, 1R-alphapinene, S-3carene, R-limonene, and alpha-terpinolene. The Monochamus galloprovincialis pheromone consists of the three main components: ipsenol, $\alpha$-pinen, and 2-methyl-3-buten-1-ol [3, 28-34]. Systematic names of pheromone molecules are given in Table 1.

\section{Results and discussion}

To analyze the effect of temperature on the pheromone structure, we divided the molecules of the chosen insects into three groups (Fig. 3) according to their structural type: acyclic (Group I), monocyclic (Group II), and bicyclic (Group III). The data obtained by calculating the temperature effect on the atomic structure of pheromone molecules were compared with the calculated data on the effect of light. To analyze the effect of temperature on the stability of different pheromone molecules, we calculated the effective frequencies of breaking of the weakest bonds in a molecule. To do this, we used Eq. (1) for calculating the probabilities of breaking of each bond in a molecule, i.e., the probabilities of the fact that the change in the bond length in a molecule exceeds the "maximum" bond extension $\mathrm{L}_{\mathrm{Max}}=0.98 \mathrm{~A}$.

Table 1 (second column) gives the probabilities of breaking of the weakest bonds in a molecule under heat fluctuations for different pheromone classes. All the probabilities are normalized to the probability of breaking of the weakest bond (the probability of its breaking is 
given in parenthesis). The table data are given for the bonds with the breaking probability of no less than 0.01 of the probability of breaking of the weakest bond. The normalized probabilities of breaking of the weakest double bonds in a molecule are marked with the asterisk. The calculated temperature effect (second column) on the pheromone molecule geometry is compared with the calculated effect of light. The equilibrium lengths of the calculated weakest bonds are given in the third column.

The fifth column gives the bond length variations for the corresponding molecule after its photoexcitation. Sign + indicates the increase in the bond length after photoexcitation of a molecule; negative values indicate the decrease in the bond length. The rightmost column shows whether the bond length variations in the excited state coincide with the data of analysis of vibrations.

Table 1. Variations in the structural characteristics of molecules under temperature and photoexcitation

\begin{tabular}{|c|c|c|c|c|c|c|}
\hline \multirow{2}{*}{$\begin{array}{c}\text { Number of } \\
\text { carbon } \\
\text { atoms }\end{array}$} & \multicolumn{3}{|c|}{ Temperature excitation } & \multicolumn{2}{|c|}{ Photoexcitation } & \multirow{2}{*}{$\begin{array}{l}\text { Coincidence of } \\
\text { the calculated } \\
\text { KS and excited } \\
\text { state }\end{array}$} \\
\hline & \multicolumn{2}{|c|}{$\begin{array}{c}\text { normalized } \\
\text { (absolute) fracture } \\
\text { frequency, } \\
\text { dimensionless } \\
\text { value }\end{array}$} & $\mathrm{L}^{*}, \AA$ & ${ }^{+} \mathrm{L}_{\mathrm{ex}}, \AA$ & $\Delta \mathrm{L}^{\&}, \AA$ & \\
\hline \multicolumn{7}{|c|}{ Group I } \\
\hline \multicolumn{7}{|c|}{ 2-methyl-3-buten-2-ol } \\
\hline $2-3$ & \multicolumn{2}{|l|}{1} & 1,541 & 1,645 & $+0,104$ & coincide \\
\hline $1-2$ & \multicolumn{2}{|l|}{0,75} & 1,540 & 1,541 & $+0,001$ & not coincide \\
\hline$\#_{5-4}$ & \multicolumn{2}{|c|}{$1,958 \mathrm{e}-051$} & 1.330 & 1,445 & $+0,111$ & coincide \\
\hline \multicolumn{7}{|c|}{ 2methyl-3buten-1ol } \\
\hline $3-4$ & \multicolumn{2}{|l|}{1,00} & 1,541 & 1,533 & $-0,008$ & not coincide \\
\hline $3-5$ & \multicolumn{2}{|l|}{0,60} & 1,535 & 1,541 & $+0,006$ & not coincide \\
\hline${ }^{\#} 2-1$ & \multicolumn{2}{|c|}{$1.5618 \mathrm{e}-049$} & 1,338 & 1,404 & $+0,066$ & coincide \\
\hline \multicolumn{7}{|c|}{$\begin{array}{c}\text { *Ipsenol } \\
* * 2-M e t h y l-6-m e t h y l e n e-7-o c t e n-4-o l\end{array}$} \\
\hline $4-5$ & \multicolumn{2}{|l|}{1,00} & 1,559 & 1,551 & $-0,008$ & not coincide \\
\hline $5-6$ & \multicolumn{2}{|l|}{0,01} & 1,525 & 1,426 & $-0,099$ & coincide \\
\hline $5-7$ & 0,05 & & & 1,600 & $+0,040$ & coincide \\
\hline${ }^{\#} 22-24$ & $1.1612 \mathrm{e}-0$ & & & 1,383 & $+0,042$ & coincide \\
\hline${ }^{\#} 8-23$ & $1.2791 \mathrm{e}-0$ & & & 1,323 & $-0,022$ & coincide \\
\hline & & & & & & \\
\hline & & & & nol & & \\
\hline $7-8$ & 1 & & & & $+0,009$ & coincide \\
\hline$\#_{5-6}$ & $4.7729 \mathrm{e}-021$ & & & & $+0,033$ & coincide \\
\hline$\# 4-3$ & $3.5389 \mathrm{e}-023$ & & & & $+0,028$ & coincide \\
\hline${ }^{\#} 2-1$ & $4.7371 \mathrm{e}-023$ & & & & $+0,033$ & coincide \\
\hline & 1-M & & & $\begin{array}{l}\text { lene } \\
\text { lene)-c }\end{array}$ & exene & \\
\hline
\end{tabular}




\begin{tabular}{|c|c|c|c|c|c|}
\hline $5-7$ & 1,00 & 1,539 & 1,580 & $+0,041$ & coincide \\
\hline $4-6$ & 0,03 & 1,520 & 1,482 & $-0,038$ & not coincide \\
\hline \#8-9 & $1.6461 \mathrm{e}-046$ & 1,342 & 1,437 & $+0,095$ & coincide \\
\hline$\#_{2-4}$ & $2.7368 \mathrm{e}-042$ & 1,352 & 1,424 & $+0,072$ & coincide \\
\hline \multicolumn{6}{|c|}{$\begin{array}{c}\text { R-lemonen } \\
\text { (R)-1-Methyl-4-(1-methylethenyl)-cyclohexene }\end{array}$} \\
\hline $2-5$ & 1,00 & 1,546 & 1,542 & $-0,003$ & not coincide \\
\hline $1-2$ & 0,01 & 1,536 & 1,537 & $+0,001$ & not coincide \\
\hline$\#_{3-4}$ & $1.1358 \mathrm{e}-047$ & 1,343 & 1,419 & $+0,076$ & not coincide \\
\hline${ }^{\#} 8-19$ & $2.3943 \mathrm{e}-048$ & 1,341 & 1,447 & $+0,106$ & not coincide \\
\hline \multicolumn{6}{|c|}{ Group III } \\
\hline \multicolumn{6}{|c|}{$\begin{array}{c}\text { Trans-mytrenol } \\
\text { (1S,5R)-6,6-Dimethylbicyclo[3.1.1]heptan-2-ol }\end{array}$} \\
\hline $3-7$ & 1 & 1,574 & 1,573 & $-0,001$ & not coincide \\
\hline $1-7$ & 0,92 & 1,571 & 1,541 & $-0,030$ & not coincide \\
\hline $2-3$ & 0,01 & 1,559 & 1,557 & $-0,002$ & coincide \\
\hline $5-1$ & 0.007 & 1,540 & 1,856 & $+0,318$ & coincide \\
\hline \multicolumn{6}{|c|}{$\begin{array}{l}\text { Cis-verbenol } \\
\text { cis-4,6,6-Trimethylbicyclo[3.1.1]hept-3-en-2-ol }\end{array}$} \\
\hline $1-6$ & 1 & 1,583 & 2,633 & $+1,050$ & coincide \\
\hline \multicolumn{6}{|c|}{$\begin{array}{c}\text { Mytrenol } \\
\text { 6,6-Dimethylbicyclo[3.1.1]hept-2-en-2-yl-methanol }\end{array}$} \\
\hline $2-8$ & 1 & 1,584 & 2,646 & $+1,062$ & coincide \\
\hline $8-6$ & 0,03 & 1,577 & 1,489 & $-0,088$ & coincide \\
\hline \multicolumn{6}{|c|}{$\begin{array}{c}\text { Trans-verbenol } \\
\text { (1S,5R)-6,6-Dimethylbicyclo[3.1.1]heptan-2-ol }\end{array}$} \\
\hline $1-6$ & 1 & 1,586 & 1,670 & $+0,084$ & coincide \\
\hline$\#_{3-2}$ & $7.8559 \mathrm{e}-053$ & 1,344 & 1,434 & $+0,090$ & coincide \\
\hline \multicolumn{6}{|c|}{$\begin{array}{c}\text { Alpha-pinene } \\
\text { 2,6,6-Trimethylbicyclo[3.1.1]hept-2-ene }\end{array}$} \\
\hline $6-7$ & 1 & 1,583 & 2,777 & $+1,194$ & coincide \\
\hline${ }^{\#} 4-5$ & $8.8800 \mathrm{e}-053$ & 1,344 & 1,421 & $+0,077$ & not coincide \\
\hline \multicolumn{6}{|c|}{$\begin{array}{c}\text { 1R-alpha-pinene } \\
(1 \mathrm{R}, 5 \mathrm{R})-2,6,6-\mathrm{Trimethylbicyclo[3.1.1]hept-2-ene}\end{array}$} \\
\hline $1-2$ & 1,00 & 1,583 & 2,721 & $+1,138$ & coincide \\
\hline $1-4$ & 0,01 & 1,576 & 1,485 & $-0,091$ & not coincide \\
\hline \multicolumn{6}{|c|}{$\begin{array}{c}\text { 1S-alpha-pinene } \\
\text { (1S)-2,6,6-Trimethylbicyclo[3.1.1]hept-2-ene }\end{array}$} \\
\hline $5-6$ & 1,00 & 1,583 & 2,835 & $+1,252$ & coincide \\
\hline $4-6$ & 0,02 & 1,576 & 1,480 & $-0,096$ & coincide \\
\hline \multicolumn{6}{|c|}{$\begin{array}{l}\text { 3S-caren } \\
\text { (1S)-3,7,7-Trimethylbicyclo[4.1.0]hept-3-ene }\end{array}$} \\
\hline $3-6$ & 1,00 & 1,517 & 1,825 & $+0,308$ & coincide \\
\hline $3-7$ & 0,04 & 1,523 & 1,499 & $-0,024$ & coincide \\
\hline $5-4$ & 0,02 & 1,545 & 1,543 & $-0,002$ & coincide \\
\hline $6-7$ & 0,08 & 1,524 & 1,505 & $-0,019$ & coincide \\
\hline
\end{tabular}


\# is double bond,

+ is the increase in the bond length under photoexcitation, and

- is the decrease in the bond length under photoexcitation;

$\mathrm{L}^{*}, \AA$ is the bond length in the ground state in angstrom;

${ }^{+} \mathrm{L}_{\mathrm{ex}}, \AA$ is the bond length in a molecule in the excited state in angstrom;

$\Delta \mathrm{L}^{\boldsymbol{\&}}, \AA$ is the difference between the bond lengths in the ground and excited states in angstrom.

Analysis of the vibrational modes allowed us to estimate the role of temperature in the breaking of chemical bonds. The calculated excited state of molecules makes it possible to characterize the effect of solar radiation on the pheromone structure. The group-I acyclic molecules 2-methyl-3buten-2-ol (Fig. 3 I-A, I-B) and 2-methyl-3-buten-1-ol (Fig. 3 I-C, I-D) contain one double bond each; the strongest structural variations under excitation of molecules are observed in the multiple bond region. In the ipsenol molecule (Fig. 3 I-E, I-F) containing a system of conjugated double bonds, the structural changes in the multiple bond region under excitation are not stronger than the changes in the single bond length. As expected, the Hessian analysis did not show significant vibrations of double bonds, unlike the case of single bonds. Therefore, upon vibrations, the bonds with the maximum length can easily break. In the 2methyl-3-buten-1-ol molecule, the change in the $\mathrm{C} 2-\mathrm{C} 3$ bond length comparable with the change in the double bond length coincides with the place of the most probable structural changes in the analysis of vibrations (Table 1). In the ipsenol molecule, the strongest changes in the length of single bonds in the structure also coincided with the calculated Hessian data, although the probability of variations in the $\mathrm{C} 5-\mathrm{C} 6$ and $\mathrm{C} 5-\mathrm{C} 7$ bond lengths is lower than the probability of variations in the C4-C5 bond length (Table 1). 

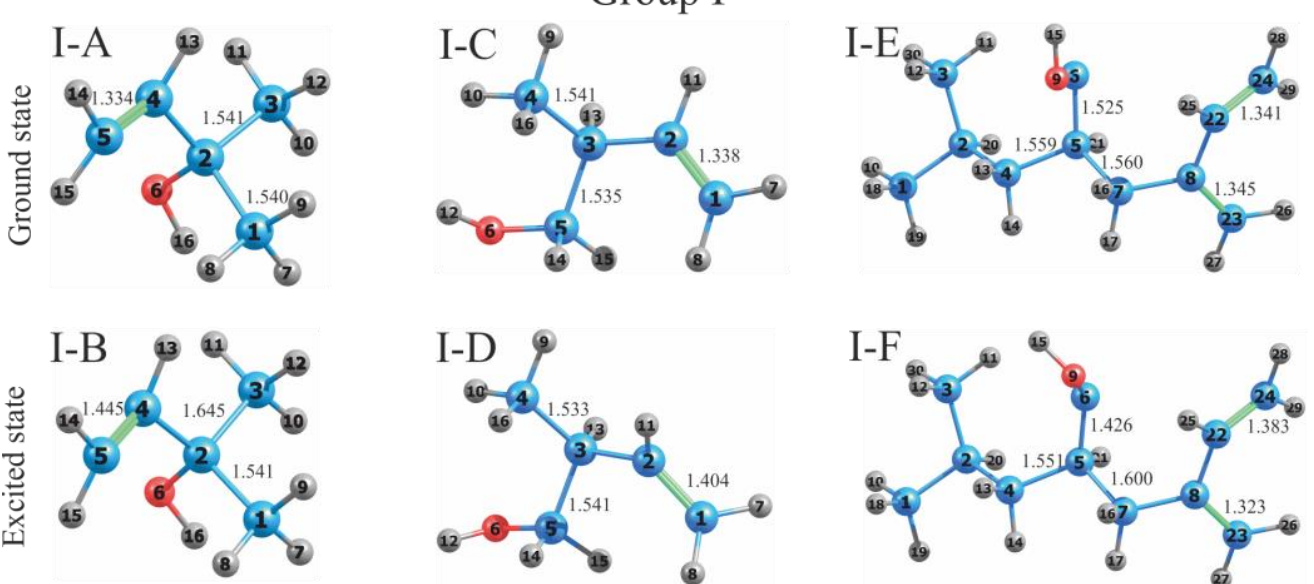

2-methyl-3-buten-2ol

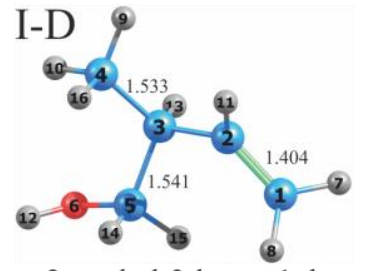

2-methyl-3-buten-1ol
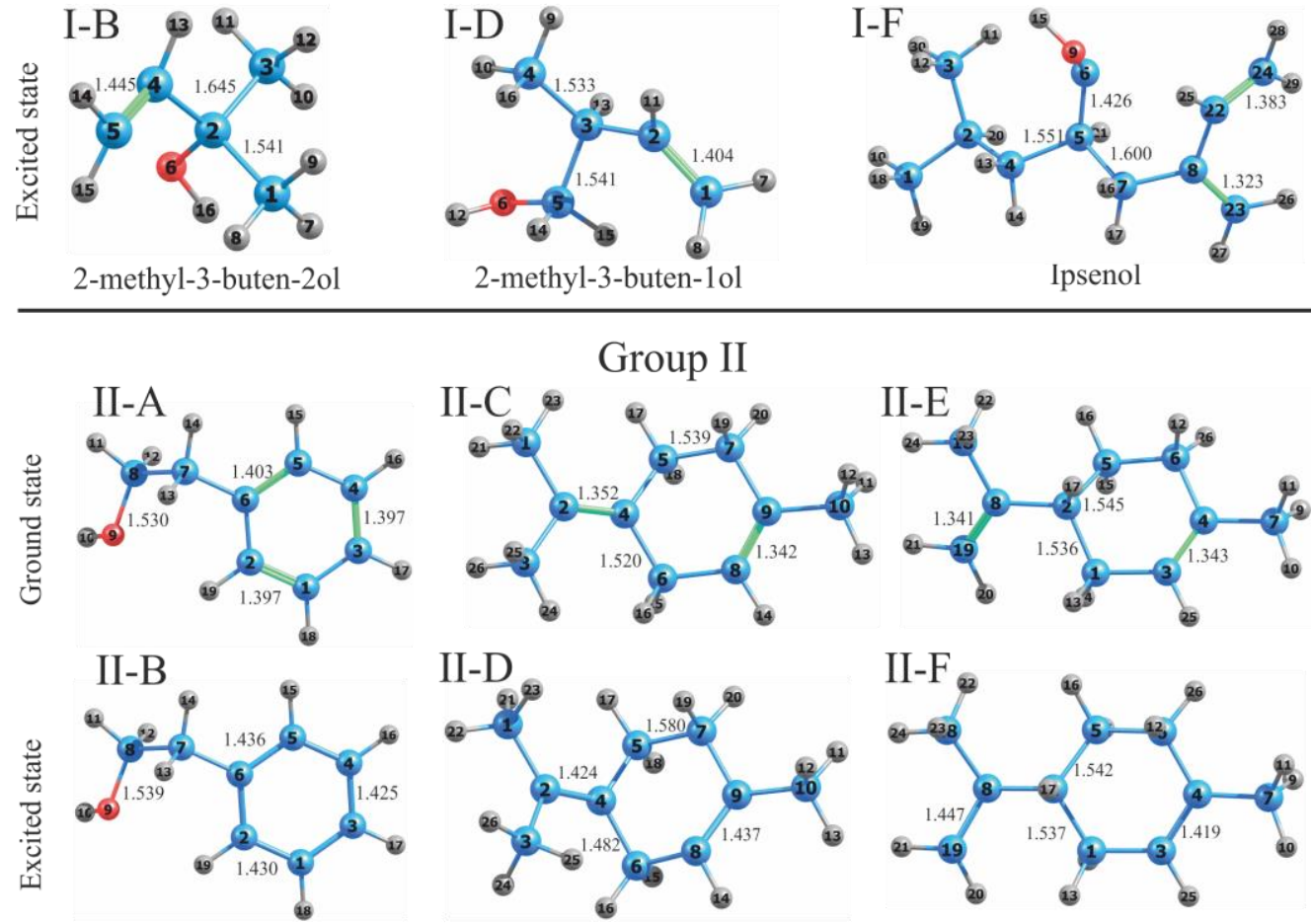

2-phenyl-ethanol

Alpha-terpinolene

R-lemonen

Group III
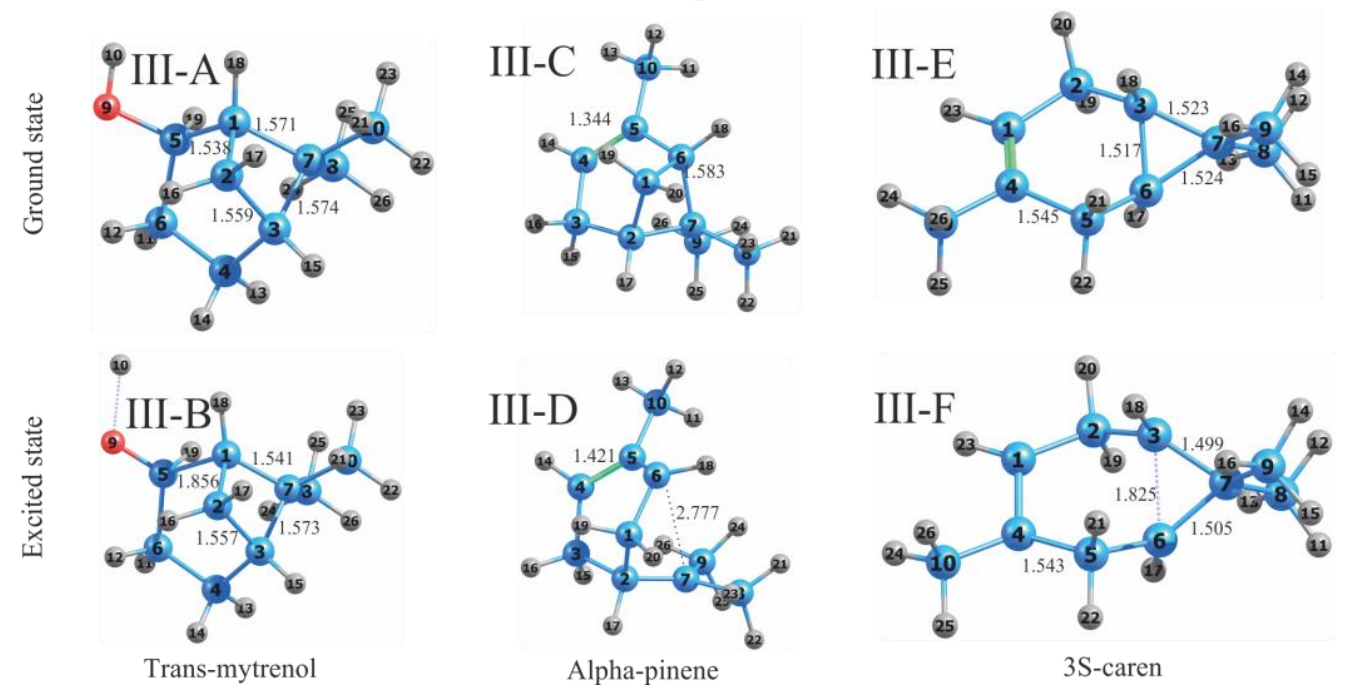

Fig. 3 Atomic structure of xylophage pheromone molecules in the ground and excited states. Group 1 includes acyclic molecules; group 2, monocyclic molecules; and group 3, bicyclic molecules. Carbon atoms are marked in blue, oxygen atoms in red, hydrogen atoms in gray 
Group 2 includes monocyclic pheromone molecules. In the 2-phenyl-ethanol molecule, the strongest change in the bond length under excitation is observed between atoms C7-C8 (Fig. 3 II-A, II-B); the temperature effect also changes the C7-C8 bond the most (Table 1). After absorption of the light quantum, the bonds inside the benzene ring of the 2-phenyl-ethanol molecule slightly change (Fig. 3 II-B), while the temperature excitation does not affect the benzene ring. In alpha-terpinolene (Fig. 3 II-C, II-D) containing two isolated double bonds, both the photo- and temperature excitations change the length of the single $\mathrm{C} 5-\mathrm{C} 7$ and $\mathrm{C} 4-\mathrm{C} 6$ bonds included in the hexatomic cycle. The $\mathrm{C} 5 \mathrm{-C} 7$ bond increases by $0.041 \mathrm{~A}$ and the $\mathrm{C} 4-\mathrm{C} 6$ bond decreases by $0.038 \mathrm{~A}$ (Table 1). The strongest change in the bond length after absorption of light is observed for the double $\mathrm{C} 2-\mathrm{C} 4$ bond, which is not included in the cycle (Fig. 3 II-D). In the Rlemonen molecule (Fig. 3 II-E, II-D), the temperature excitation will, most probably, lead to the change in the lengths of single $\mathrm{C} 2-\mathrm{C} 5$ and $\mathrm{C} 1-\mathrm{C} 2$ bonds included in the cycle, while the photoexcitation does not change the length of these bonds (Table 1). Absorption of light leads to the extension of double bonds by $0.076 \mathrm{~A}$ inside the cycle and by $0.106 \mathrm{~A}$ beyond it. Similar to the case of acyclic pheromone molecules, the absorption of light in cyclic molecules changes most strongly the structure of multiple bonds, while the heat radiation affects single bonds.

Group 3 consists of bicyclic pheromone molecules. Figure 3 shows only three molecules of this type. Since the structural variations in the alpha-pinene, cis-verbenol, mytrenol, transverbenol, 1R-alpha-pinene, and 1S-alpha-pinene molecules under irradiation are similar, Figure 3 only shows the alpha-pinene molecule.

The thermal vibrations in the trans-mytrenol molecule (Fig. 3 III-A, III-B) will most probably lead to the breaking of the $\mathrm{C} 3-\mathrm{C} 7$ andC7-C1 bonds included in both the tetratomic and hexatomic cycle. Since the initial C3-C7 bond length is $1.573 \mathrm{~A}$ and the $\mathrm{C} 1-\mathrm{C} 7-\mathrm{C} 3$ angle is $85^{\circ}$ instead of the angle $109^{\circ}$ typical of the $\mathrm{sp}^{3}$-hybridized orbitals, we may assume that the change in the bond length is related to the breaking of one of the bonds and opening of the strained tetratomic cycle. Upon radiation absorption, the $\mathrm{C} 5-\mathrm{C} 1$ bond included only in the hexatomic cycle extends by 0.318 A. Photoexcitation will, most likely, lead to the opening of the hexatomic cycle, while the tetratomic cycle remains invariable, in contrast to the case of the temperature effect (Table 1).

In the alpha-pinene (Fig. 3 III-C, III-D), cis-verbenol, mytrenol, trans-verbenol, 1Ralpha-pinene, and 1S-alpha-pinene molecules, the maximum elongations of a single bond included in the hexatomic and tetratomic cycle under the temperature and photoexcitation are the same (Table 1), despite the presence of double bonds in the structure. The geometry of molecules in the excited state is indicative of the possibility of opening of the strained tetratomic cycle with the retained hexatomic cycle in the structure (Fig. 3 III-C, III-D). The breaking of the single 
bond at the opening of the cycle without additional interaction with other substances (air components) can lead to the electron density redistribution and formation of a double bond in the monocyclic molecule.

The calculation showed that in 3S-caren (Fig. 3 III-E, III-F), both the photo- and temperature excitations lead to the lengthening of the C3-C6 bond included in the hexatomic and triatomic cycles (Table 1). The bond length increases under photoexcitation by $0.308 \mathrm{~A}$, which is noticeably smaller than in the molecules with the tetratomic and hexatomic cycles. At the opening of the tetratomic cycle in the previously investigated compounds, the hexatomic cycle was retained, while the breaking of the C3-C6 bond in 3S-caren should lead to the formation of the heptatomic cycle in the excited state (Fig. 3 III-F). The atomic structure of a molecule in the excited state can return to the initial state; therefore, despite the growth of the C3-C6 bond length, the initial structure will, most probably, be retained.

Due to the presence of strained cycles [35], many terpene compounds tend to the thermal isomerization. The temperature transformations are accompanied by the opening of the tetratomic cycle or tetratomic and hexatomic cycles simultaneously. Figure $4 \mathrm{~B}$ shows a schematic of the thermal decomposition [35] and Fig. 4 A, a calculated schematic of the atomic structure variation.
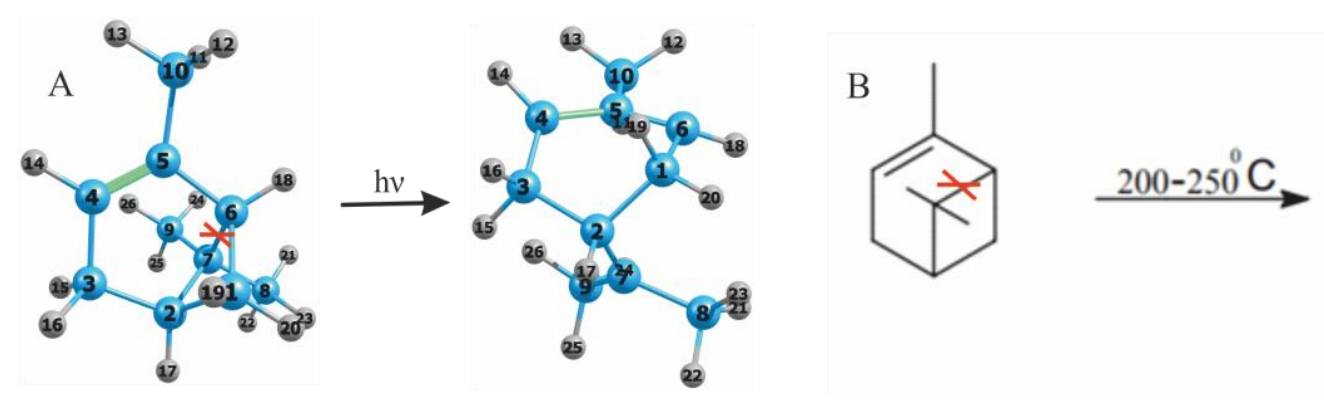

Fig. 4 Isomerization of the alpha-pinene molecule: (A) calculated data and (B) data from [35]

According to the calculation, in the alpha-pinene molecule both under the photo- and temperature excitation, the single C6-C7 bond length increases (under photoexcitation, by 1.194 A). Thus, the experimental data on the thermal isomerization of a molecule caused by the bond breaking and opening of the tetratomic cycle are consistent with the data obtained using the technique of determination of the KS of molecules at high temperatures.

\section{Conclusions}


Analysis of the terpene molecules is of great importance for ecology, since these molecules represent pest insect pheromones, and, in addition, can be used in perfumery industry. Using the quantum chemical methods, we studied the excited state and calculated the bond vibrations for the organic molecules containing the strained cycles and conjugated, double, and single bonds. We estimated the effect of temperature on the atomic structure by the kinetic stability technique. The approaches used allowed us to estimate the stability of molecules against solar radiation and temperature. The temperature excitation of the unsaturated acyclic and monocyclic compounds will, most probably, change the single bond lengths, while the photoexcitation affects first the multiple bonds and leads to their elongation. In the unsaturated bicyclic terpenes, the photo- and temperature excitations lead to the analogous structural transformations, specifically, to the probable breaking of the single bond included in both cycles and opening of the tetratomic cycle at the retained hexatomic one. The analogous thermal isomerization reaction in the alpha-pinene molecule was described in the literature, which confirms the reliability of the calculated data. Thus, there are two possible parallel mechanisms of deactivation of the calculated molecules: under solar radiation and under the action of temperature.

Our analysis can be interesting for ecological application, since it allows, first, estimating the stability of pheromones and, second, predicting the features of chemical communication of insects. Since for the species searching for a mate at night time, i.e., at low temperatures and minimum electromagnetic radiation intensity, the pheromone molecules can start decomposing in the beginning of the daylight under the action of, i.e., UV radiation and continue decomposing with an increase in the air temperature during the day, the concentration of molecules by the next night will be minimum. The characteristic lifetime of pheromone molecules of the insects searching for a mate during the daytime, i.e., at the strong electromagnetic radiation and high air temperature, can be short. This can limit the radius of attraction of mates to the insects producing pheromones. Thus, the characteristic lifetime of pheromone molecules will determine the mechanism of pheromone search and the type of molecules that can be used as pheromones.

\section{Acknowledgments}

This study was supported by the Russian Foundation for Basic Research, Government of the Krasnoyarsk Territory, and the Krasnoyarsk Territorial Foundation for Support of Scientific and R\&D Activities, project no. 16-44-243019 and the Russian Foundation for Basic Research, project no. 15-02-06869 and no. 16-04-00132. 


\section{References}

1. Regnier FE, Law JH (1968) Insect pheromones. J Lipid Res. 9 (5): 541-551.

2. Buser H-R, Guerin PM, Tóth M, Szöcs G, Schmid A, Francke W and Arn H (1985) (Z,Z)-6,9-nonadecadien-3-one and (Z,Z,Z)-3,6,9-nonadecatriene: identification and synthesis of sex pheromone components of Peribatodes rhomboidaria. Tetrahedron Lett. 26:403406.

3. Pajares J.A., Alvarez G, Ibeas F et al (2010) Identification and Field Activity of a Male-Produced Aggregation Pheromone in the Pine Sawyer Beetle, Monochamus galloprovincialis. J. Chem. Ecol. 36 (6): 570-583.

4. Lei H, Chiu H-Y (2013) Responses of protocerebral neurons in Manduca sexta to sex-pheromone mixtures. Journal of Comparative Physiology A. 199 (11): 997-1014.

5. Tomilin FN, Osina OV, Kuzubov AA et al (2011) Stability of forest lepidopteran pheromones against environmental factors. Biophysics 4: 714-722.

6. Shi R-W, Liu F (2016) Quantum chemical study on the stability of honeybee queen pheromone against atmospheric factors. J Mol Model. 22:140.

7. Soukhovolsky VG, Tomilin FN, Artyushenko PV and Tsikalova PE (2016) System of Forest Insect Pheromone Communication: Stability of «Information» Molecules to Environmental Factors. Siberian Journal of Forest Science 3: 67-76.

8. Soukhovolsky VG, Volkova (Tsikalova) PE, Tarasova OV (2014) Modeling of Forest Insect Pheromone Communication System. Female as a Source of Information,Siberian. Journal of Forest Science 6: 69-79.

9. Artyushenko PV, Tomilin FN, Ovchinnikov SG et al (2017) The stability of the pheromones of xylophagous insects to environmental factors: An evaluation by quantum chemical analysis. Biophysics 62: 532-538.

10. Fedorov AS, Fedorov DA, Kuzubov AA, Avramov PV, Nishimura Y, Irle S and Witek HA (2011) Relative Isomer Abundance of Fullerenes and Carbon Nanotubes Correlates with Kinetic Stability. Phys.Rev.Lett.107: 175506.

11. Fedorov AS, Kuzubov AA, Visotin MA, Tomilin FN (2017) New method for calculations of nanostructure kinetic stability at high temperature, Journal of Magnetism and Magnetic Materials, 440: 167-170.

12. Lee C, Yang W and Parr RG (1988) Development of the Colle-Salvetti correlation-energy formula into a functional of the electron density. Phys. Rev. B 37: 785-789.

13. Becke AD (1988) Density-functional exchange-energy approximation with correct asymptotic behavior. Phys. Rev. A 38: 3098-3100. 
14. Miehlich B, Savin A, Stoll H, Preuss H (1989) Results obtained with the correlation energy density functionals of Becke and Lee-Yang and Parr. Chem Phys Lett. 157: 200-206.

15. Schmidt MW, Baldridge KK, Boatz JA, Elbert ST, Gordon MS, Jensen JH, Koseki S and Matsunagaetal N (1993) General Atomic and Molecular Electronic Structure System. Comput. Chem. 14: 1347-1363.

16. Runge E and Gross EKU (1984) Density-Functional Theory for Time-Dependent Systems. Phys. Rev. Lett. 52-12: 997-1000.

17. Petersilka M, Gossmann U, and Gross EKU (1996) Excitation Energies from Time-Dependent Density-Functional Theory. Phys. Rev. Lett. 76-8: 1212-1215.

18. Curtiss LA, Raghavachari K, Redfern PC and Pople JA (1997) Assessment of Gaussian-2 and density functional theories for the computation of enthalpies of formation. $\mathrm{J}$. Chem. Phys. 106 (3): 1063- 1068.

19. Curtiss LA, Redfern PC and Raghavachari K (2005) Assessment of Gaussian-3 and test set of experimental energies. J. Chem. Phys. 123: 124107-124119.

20. Tirado-Rives J and Jorgensen WL (2008) Performance of B3LYP Density Functional Methods for a Large Set of Organic Molecules. J. Chem. Theory Comput. 4: 297306.

21. Fredj D, Hassen CB, Elleuch S, Feki H, Boudjada NC, Mhiri T and Boujelbene M (2017) Structural, vibrational and optical properties of a new organic-inorganic material: (C5H8N3)2[BiCl5], J. Mater. Res. Bull. 85: 23-29.

22. Schuchardt KL, Didier BT, Elsethagen T, Sun L, Gurumoorthi V, Chase J, Li J and Windus TL (2007) Basis Set Exchange: A Community Database for Computational Sciences. J. Chem. Inf. Model. 47-3: 1045-1052.

23. Feller D (1996) The role of databases in support of computational chemistry calculations. J. Comp. Chem. 17-13: 1571- 1586.

24. Artyushenko PV, Tomilin FN, Kuzubov AA, Ovchinnikov SG, Tsikalova PE, Ovchinnikova TM, Soukhovolsky VG (2016) Action of the atomic and electronic structure of pheromone molecules on the effectiveness of communication in xylophagous insects. J. Structural Chemistry 57 (2): 287- -293.

25. Nesmeyanov AN and Nesmeyanov NA (1974) Organic chemistry. Chemistry, Moscow.

26. Kirk RE and Othmer DF (1983) Encyclopedia of Chemical Technology 22, 3rd Ed. John Wiley \& Sons, New York. 
27. Breitmaier E (2006) Terpenes: Flavors, Fragrances, Pharmaca, Pheromones. Willey-VCH Verlag GmbH \& Co. KGaA, Weinheim.

28. Birgersson G, Schlyter F, Löfqvist J et al (1984) Quantitative variation of pheromone components in the spruce bark beetle Ips typographus from different attack phases. J. Chem. Ecol. 10: 1029-1055.

29. Pettersson E, Boland W (2003) Potential parasitoid attractants, volatile composition throughout a bark beetle attack. Chemoecology 13: 27-37.

30. Fan J, Kang L, Sun J (2007) Role of host volatiles in mate location by the Japanese pine sawyer, Monochamus alternatus hope (Coleoptera: Cerambycidae). Environ Entomol. 36: 58-63.

31. Allison JD, Borden JH, McIntosh RL et al (2001) Kairomonal response by four Monochamus species (Coleoptera: Cerambycidae) to bark beetle pheromones. J. Chem. Ecol. 27: 633-647.

32. Nabil N (2009) State of the Art on the Monitoring of the Pine Wood Nematode PWN (Bursaphelenchusxylophilus) and Its Insect Vector (Monochamusgalloprovincialis) in Europe. Institut Européen de la forêtcultivée EFIATLANTIC, Cestas.

33. Ibeas F, Diez JJ and Pajares JA (2008) Olfactory sex attraction and mating behaviour in the pine sawyerMonochamus galloprovincialis (Coleoptera: Cerambycidae). J. Insect Behav. 21: 101-111.

34. Ibeas F, Gallego D, Diez JJ et al (2007) An operative kairomonal lure for managing pine sawyer beetle Monochamus galloprovincialis (Coleoptera: Cerymbycidae). J. Appl. Entomol. 131: 13-20.

35. Plemenkov VV (2007) Khimiya izoprenoidov (The Chemistry of Isoprenoids). Izd. Altais. Univer., Barnaul. 